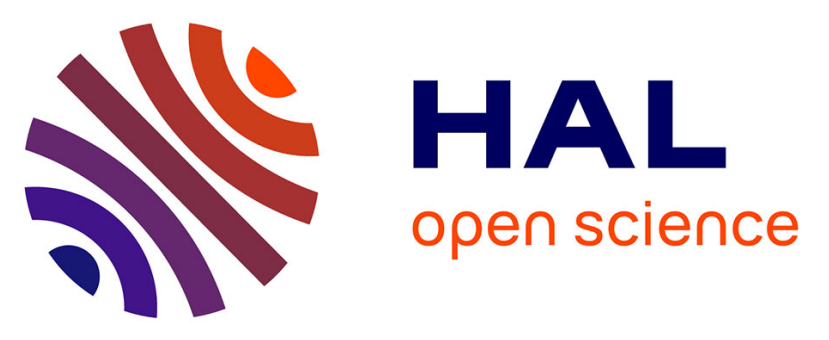

\title{
Enhanced performance of integrated silicon nanophotonic devices engineered with sub-wavelength grating structures
}

\author{
Daniel Benedikovic, Mathias Berciano, Alberto Carlos, Xavier Le Roux, \\ Sylvain Guerber, Guillaume Marcaud, Vladyslav Vakarin, Diego \\ Perez-Galacho, Eric Cassan, Delphine Marris-Morini, et al.
}

\section{To cite this version:}

Daniel Benedikovic, Mathias Berciano, Alberto Carlos, Xavier Le Roux, Sylvain Guerber, et al.. Enhanced performance of integrated silicon nanophotonic devices engineered with sub-wavelength grating structures. SPIE Optics + Optoelectronics 2019 Integrated Optics: Design, Devices, Systems, and Applications V, Apr 2019, Prague, Czech Republic. 10.1117/12.2520780 . hal-02539514

\author{
HAL Id: hal-02539514 \\ https://hal.science/hal-02539514
}

Submitted on 10 Apr 2020

HAL is a multi-disciplinary open access archive for the deposit and dissemination of scientific research documents, whether they are published or not. The documents may come from teaching and research institutions in France or abroad, or from public or private research centers.
L'archive ouverte pluridisciplinaire HAL, est destinée au dépôt et à la diffusion de documents scientifiques de niveau recherche, publiés ou non, émanant des établissements d'enseignement et de recherche français ou étrangers, des laboratoires publics ou privés. 


\title{
Enhanced performance of integrated silicon nanophotonic devices engineered with sub-wavelength grating structures
}

\author{
Daniel Benedikovic a, ${ }^{\text {, }}$, Mathias Berciano ${ }^{\mathrm{a}}$, Carlos Alberto Alonso-Ramos ${ }^{\mathrm{a}}$, Xavier Le Roux ${ }^{\mathrm{a}}$, Sylvain \\ Guerber $^{\mathrm{a}, \mathrm{b}}$, Guillaume Marcaud ${ }^{\mathrm{a}}$, Vladyslav Vakarinn, Diego Pérez-Galacho ${ }^{\mathrm{a}, \mathrm{c}}$, Eric Cassan ${ }^{\mathrm{a}}$, \\ Delphine Marris-Morini ${ }^{\mathrm{a}}$, Pavel Cheben ${ }^{\mathrm{c}}$, Frédéric Boeuf ${ }^{\mathrm{b}}$, Charles Baudot ${ }^{\mathrm{b}}$, and Laurent Vivien ${ }^{\mathrm{a}}$
}

\author{
${ }^{a}$ Centre de Nanoscience et de Nanotechnologies, CNRS, University of Paris-Sud, Université Paris- \\ Saclay, C2N - Palaiseau, 91120 Palaiseau, France \\ ${ }^{b}$ Technology R\&D, STMicroelectronics SAS, 850 rue Jean Monnet - 38920 Crolles, France \\ 'Photonics Research Labs, iTEAM Research Institute, Universitat Politecnic de Valencia, Spain \\ dNational Research Council Canada, 1200 Montreal Road, Ottawa, Ontario, K1A0R6, Canada
}

\begin{abstract}
Sub-wavelength gratings, segmented resonant-less structures with geometries featuring scales considerably smaller than the wavelength of light, have enabled an attractive technological concept to locally control light guiding properties in planar silicon chip architectures. This concept has allowed for additional degrees of freedom to tailor effective mode index, modal confinement, waveguide dispersion, as well as anisotropy, thereby providing a vital route towards high performing devices with engineered optical properties. Sub-wavelength integrated nanophotonics has opened up new horizons for realization of key building components that afford outstanding device performances, typically beyond those achieved by conventional design strategies, yet favorably benefiting from the sub-100-nm pattern resolution of established semiconductor manufacturing tools in nanophotonic foundries. The distinctive features of sub-wavelength grating structures are considered essential for future generation of chip-scale applications in optical communications and interconnects, biomedicine, as well as quantum-based technologies. In this work, we report recent advances in the development of high-performance on-chip nanophotonic waveguides and devices engineered with the sub-wavelength grating metamaterial structures. In particular, we discuss recent achievements of low-loss waveguides with controlled chromatic dispersion, high-efficiency fiber-to-chip surface grating couplers, micro-ring resonators, and grating-assisted waveguide filters, implemented on the mature silicon-on-insulator technology.
\end{abstract}

Keywords: Integrated optics, Silicon nanophotonics, Sub-wavelength grating metamaterials, Waveguide dispersion, Micro-ring resonators, Fiber-chip surface grating couplers, Waveguide-based grating filters

*corresponding author: daniel.benedikovic@c2n.upsaclay.fr

\section{INTRODUCTION}

Silicon-on-insulator (SOI) has been established as a compelling waveguide system for silicon nanophotonics to realize compact components with nano-scaled dimensions. Silicon nanophotonics is worthily considered to be a key technology in future to develop optical chips with a high density of device integration. The imminent success of silicon nanophotonics is in part reached due to the compatibility with the established fabrication tools, techniques, and procedures, as developed over years by mature micro-electronics industry. Silicon nanophotonics is promising for a variety of emerging nanophotonic applications, including optical communications, intra- and inter-chip interconnects, sensing, or health and medicine, to name just a few. Most recently, the manufacturing services of silicon nanophotonic foundries has become widely accessible to the community via open-access shuttles and chip sharing initiatives. This opens up a further pathway for the fabless model development of complex integrated circuits that may contain large number of different components on a single chip. This way, silicon nanophotonic chips hold promises to afford even complex optical functionalities, beginning with light emission and modulation, through the light processing and transmitting, and finishing with light detection ${ }^{1-10}$. 
However, silicon nanophotonics also suffers from a serious shortcoming, which is a limited design space. More specifically, silicon nanophotonics offers only a limited set of fundamental materials and waveguide platforms that can be straightforwardly processed by the established foundries ${ }^{10}$. This includes materials such as silicon ( $\mathrm{Si}$ ), silicon dioxide $\left(\mathrm{SiO}_{2}\right)$, silicon nitride's ( $\mathrm{SiN}$ 's), and germanium $(\mathrm{Ge})$. The strong benefit of this group of fundamental nanophotonic materials is the fact that they can be monolithically integrated on silicon chips, however, still providing only a relatively small freedom to make a significant difference. On the other hand, integration of epitaxial III-V hetero-structures is even more challenging and comparatively complicated ${ }^{11,12}$. Furtheremore, the development of complex silicon nanophotonic devices and components calls upon multi-layer structuration's or multi-level etchings ${ }^{13,14}$. However, this comes hand-byhand with an additional fabrication and pre- or post-processing steps, which may be difficult to implement in some situations. Restrictions in requirements for minimum feature sizes, mask-layer misalignments, and etching depth variations are also more apparent. Last, but not least, inclusion of novel, typically exotic materials may move the fabrication out from the well-developed silicon nanophotonics manufacturing conventions ${ }^{10-14}$.

To address the problem of limited design space, segmenting the conventional silicon waveguides at scales substantially smaller than the wavelength of light propagating through it has been recognized as an excellent solution ${ }^{15,16}$. Such a segmented waveguide with a periodic arrangement of silicon blocks, so-called sub-wavelength grating (SWG), avoids formation of Bragg reflection zones as well as frustrates diffraction effects ${ }^{17}$. This SWG-structured waveguide acts as a homogeneous slab waveguide with an optically equivalent material, i.e. SWG metamaterial waveguides. The geometry of SWG waveguide segments offers accurate control of all critically important light propagation properties. This includes direct tailoring of material and effective index, modal confinement, field profile, dispersion, and anisotropy. The recent advances in SWGs fundamentals encompass exploitation of the SWG waveguide anisotropy ${ }^{18,19}$, reducing sources of back-scatterings ${ }^{20}$, mitigating the parasitic leakage loss towards silicon substrate ${ }^{21}$, as well as controlling jitter effects $^{22}$. Over last years, SWG structures have been identified as an important design tool to develop high-performing integrated silicon nanophotonic devices. Profound examples of superior SWG-tailored nanophotonic devices include, but not limited to, highly efficient fiber-to-chip edge ${ }^{15,16,23-26}$ and surface grating couplers ${ }^{27-38}$, broadband directional couplers $^{39,40}$ and multimode interference beamsplitters ${ }^{18}$, highly selective grating filters ${ }^{41-44}$, high quality factors $(\mathrm{Q}-$ factor) ring resonators for sensing ${ }^{45-50}$, dispersion engineered SWG metamaterial waveguides ${ }^{51,52}$, optical modulator ${ }^{53}$, or devices for extended mid-infrared operations $\mathrm{s}^{54-56}$. For more details on the theoretical background, design fundamentals, as well as on the history of SWGs in silicon nanophotonic waveguides, we refer readers to the previous extensive review articles by Halir, et al. ${ }^{57}$ or most recent summaries by Cheben, et al. ${ }^{3}$ and by Halir, et al. ${ }^{4}$.

In this work, we report on our recent advances in sub-wavelength integrated silicon nanophotonics and provide few examples of silicon waveguides and devices engineered via sub-wavelength grating nano-structuration. This particularly includes devices such as fiber-chip surface grating couplers (Section 2), SWG metamaterial micro-ring resonators (Section 3), and SWG metamaterial waveguides with an engineered chromatic dispersion (Section 4).

\section{SUB-WAVELENGTH-ENGINEERED SURFACE GRATING COUPLERS}

Efficient input / output optical interfaces are greatly recognized as essential on-chip devices in integrated silicon nanophotonics. Out-of-plane chip interfacing realized by surface grating couplers is particularly attractive solution. Grating-coupled interfaces enable flexible positioning on the chip, which is well-suited for rapid wafer-scale test assessments. Additionally, surface grating couplers are more tolerant to the alignment variations and further facilitate advanced integration and packaging strategies. However, the overall coupling efficiency of the fiber-chip grating couplers is limited by two main aspects. First, the mode size disparity between modes of standard optical fibers and submicron-sized silicon waveguides is excessively large, in particular of about two orders of magnitude. Second, significant portion of optical power is radiated towards the bottom silicon substrate, which yields in additional penalty for the overall coupling efficiency.

We proposed and experimentally demonstrated that the dual-etch fiber-chip grating couplers hold the promise to become an ideal out-of-plane coupling strategy to address the afore-mentioned concerns. Dual-etch couplers with an interleaved and an $L$-shaped waveguide geometry have been reported ${ }^{34-38}$. Exceptionally high diffraction performance, also called grating directionality, has been predicted with values typically approaching $100 \%$. This high directionality is attributed to the blazing effect as achieved via deep and shallow-etch grating trenches. This effect will ensure that the optical power radiated towards the superstrate, where the optical fiber is positioned, is maximized, while the parasitic light radiation towards bottom silicon substrate is minimized. The vertical view of the proposed $L$-shaped grating couplers is shown in Fig. 1a. The "DESIGN A" represents a nominal uniform coupler and "DESIGN B" is fundamentally identical, 
yet grating coupler has a short SWG-structured lateral pattern of silicon blocks to reduce the impact of the backreflections at the juncture between the injection waveguide and the grating coupler. Devices were implemented on 300nm-thick SOI waveguide platform and 720-nm-thick buried oxide (BOX), overcladded by silicon dioxide. The couplers were designed for operation at $1.55 \mu \mathrm{m}$ wavelength and transverse electrical (TE) polarization, with dimensions that are compatible with mass-scale deep-ultraviolet lithography. More details about the design and its unprecedented versatility can be found in Ref. [37].

According to our three-dimensional finite difference time domain (3-D FDTD) calculations, proposed $L$-shaped couplers provide a wideband diffraction performance (directionality $>90 \%$ over $100 \mathrm{~nm}$ ), as shown in Fig. 1b. It can be seen that the inclusion of the reflection-cancelling SWG transition does not affect the outstanding diffraction performance of the proposed couplers. Furtheremore, as shown in Fig. 2c, $L$-shaped couplers with a short SWG transition stage significantly decrease the grating reflectivity across the same wavelength range, with an 8 -fold reflectivity reduction at a nominal wavelength of $1.55 \mu \mathrm{m}$.

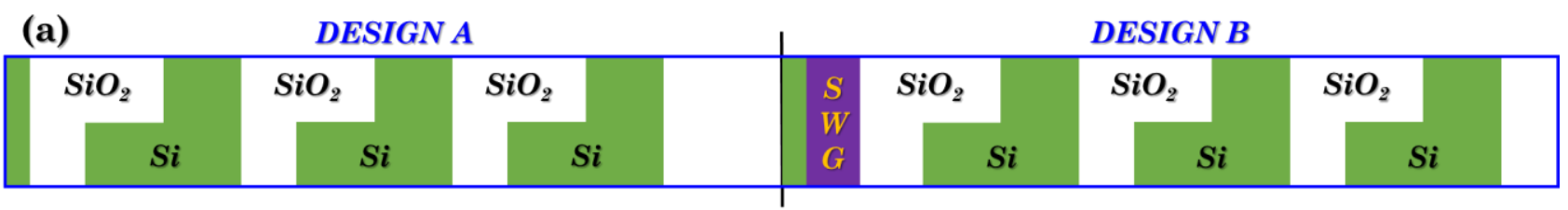

(b)

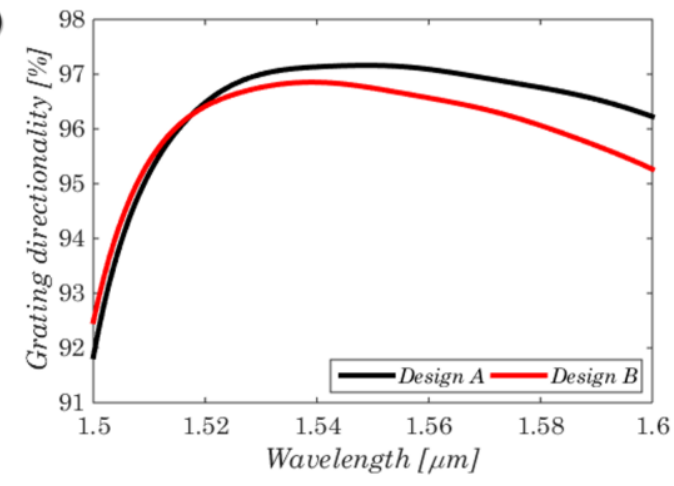

(c)

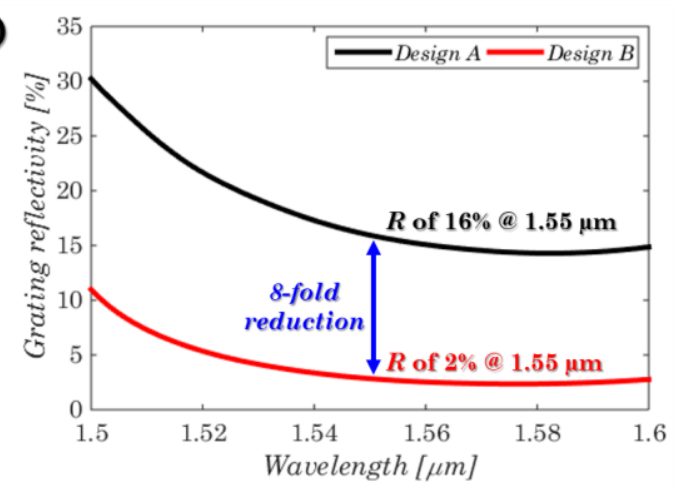

Figure 1. (a) Vertical schematics of proposed $L$-shaped fiber-chip surface grating couplers. "DESIGN $A$ " is a nominal uniform grating coupler and "DEISGN $B$ " is optimized uniform grating coupler with a short SWG transition region. Spectral evolution of (b) the grating directionality and (c) the grating reflectivity of the proposed $L$-shaped fiber-chip coupler without ("DESIGN A") and with ("DESIGN B") SWG transition region.

Figure 2 shows the calculated and the measured coupling efficiency of the proposed $L$-shaped fiber-chip grating couplers ("DESIGN A" and "DESIGN B") as a function of the wavelength. More specifically, 3-D FDTD calculations predict theoretical coupling performance up to $-2.1 \mathrm{~dB}$ for designs without and with SWG transition stage, respectively. The coupling angle for both coupler designs is $22^{\circ}$.

The $L$-shaped fiber-chip surface grating couplers were fabricated using $300 \mathrm{~mm}$ SOI photonic platform within STMicroelectronics foundry facilities. The structures were defined thanks to a 193-nm deep-ultraviolet optical lithography, followed by dual-etch process to form deep and shallow grating trenches as well as the interconnecting waveguides. Final devices were overcladded by silicon dioxide for chip protection. The scanning electron microscopy images of fabricated $L$-shaped grating coupler with a SWG transition stage are shown in the inset of Fig. 2.

Experimentally, the peak coupling loss of $-3.4 \mathrm{~dB}$ were measured for the grating coupler design without the SWG transition stage ("DESIGN A"), with a noticeable Fabry-Perot fringes observed in the retrieved spectral response. The amplitude of these fringes varies between $0.7 \mathrm{~dB}$ and $0.85 \mathrm{~dB}$. The estimated grating reflectivity is $\sim 8 \%(-11 \mathrm{~dB})$. On the other hand, the fiber-chip grating coupler with a SWG transition ("DESIGN B") provides a comparatively better coupling loss up to $-2.7 \mathrm{~dB}$. In this case, the measured spectral response also exhibits some residual fringes, yet with substantially smaller amplitude of about $\sim 0.1 \mathrm{~dB}$. This provides an estimated grating reflectivity of only $\sim 1 \%(-20 \mathrm{~dB})$. 


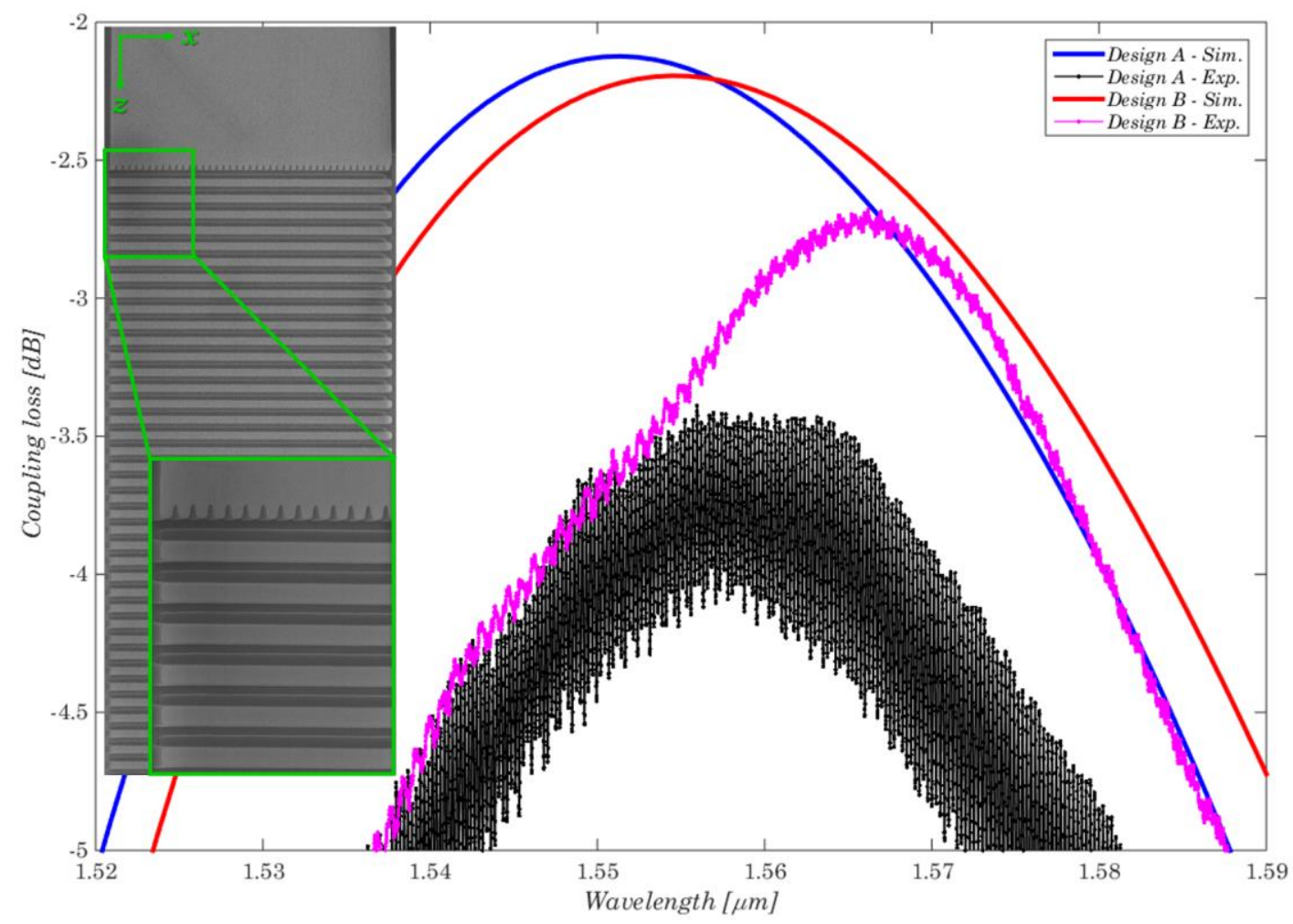

Figure 2. The calculated and the measured coupling loss of the proposed $L$-shaped fiber-chip grating couplers as a function of the wavelength. Solid lines (blue and red colors) corresponds to the 3-D FDTD design predictions, while solid lines with dots (black and magenta colors) corresponds to the experimental results. Inset: Scanning electron microscopy image of the fabricated L-shaped grating coupler with a short SWG transition stage.

For a future work in this direction, we foresee that the implementation of SWG apodization may be advantageously used to further improve the fiber-chip coupling performance, which also allows us to fully exploit the great potential of the proposed $L$-shaped fiber-chip grating couplers.

\section{SUB-WAVELENGTH METAMATERIAL MICRO-RING RESONATORS}

The SWG waveguide structures provide an additional degree of freedom to control the modal confinement of the optical field. This can be easily performed by optimizing the duty cycle of the segmented waveguide core, i.e. by tailoring the amount of silicon inside the grating structure. By lowering (enlarging) the grating duty cycle, the equivalent waveguide core index decreases (raises), and thereby the optical mode is more delocalized (more confined) from (in) the metamaterial waveguide core. This superior feature of the SWG metamaterial waveguides can be beneficially exploited to lower the level of back-scattering from SWG waveguides as compared to the conventional silicon strip waveguides ${ }^{20}$. In addition, the beneficial effect of optical mode delocalization from the segmented waveguide core is also essential for developing efficient fiber-chip couplers ${ }^{23-26}$ as well as for exploitation in on-chip evanescent field sensing applications ${ }^{45-}$ 50.

SWG waveguides as combined with a micro-ring waveguide resonators have been demonstrated, providing a significant contributions to the development of SWG-assisted on-chip biosensors ${ }^{46-50}$. However, the optical mode delocalization from the SWG waveguide core makes it challenging to realize tight bends with a reasonably low radiation losses. To this end, SWG micro-ring resonators are very attractive to enhanced light-matter interactions, however, suffering from excessively large bending losses. Figures $3 \mathrm{a}-3 \mathrm{c}$ show the spectral responses and detailed resonance views of the SWG micro-ring resonators with ring radii of $10 ; 20$; and $30 \mu \mathrm{m}$. The SWG waveguide has a period of $200 \mathrm{~nm}$ and duty cycle of 50\%. Devices were fabricated by electron beam lithography, followed by dry etching to define a waveguide features, and deposition of PMMA cladding to protect the sample. Figures $3 \mathrm{~d}$ and $3 \mathrm{e}$ show scanning electron microscopy images of fabricated devices. As can be observed, in all cases very high extinction ratio of about $-30 \mathrm{~dB}$ was obtained. In this 
example, the corresponding quality factors were determined to be $1700(R=10 \mu \mathrm{m}), 9200(R=20 \mu \mathrm{m})$, and $14000(R=$ $30 \mu \mathrm{m})$, respectively. Here, quality factors increase with larger ring radii due to the reduced bending losses. Significant improvement of the quality factors of SWG ring resonators can be achieved by using trapezoidally-shaped silicon blocks for the curved waveguides ${ }^{50}$. Mode index is engineered inside the waveguide itself, i.e. the local effective mode index is gradually changed across the waveguide width to achieve mode shifting and thereby decreasing the bending losses.
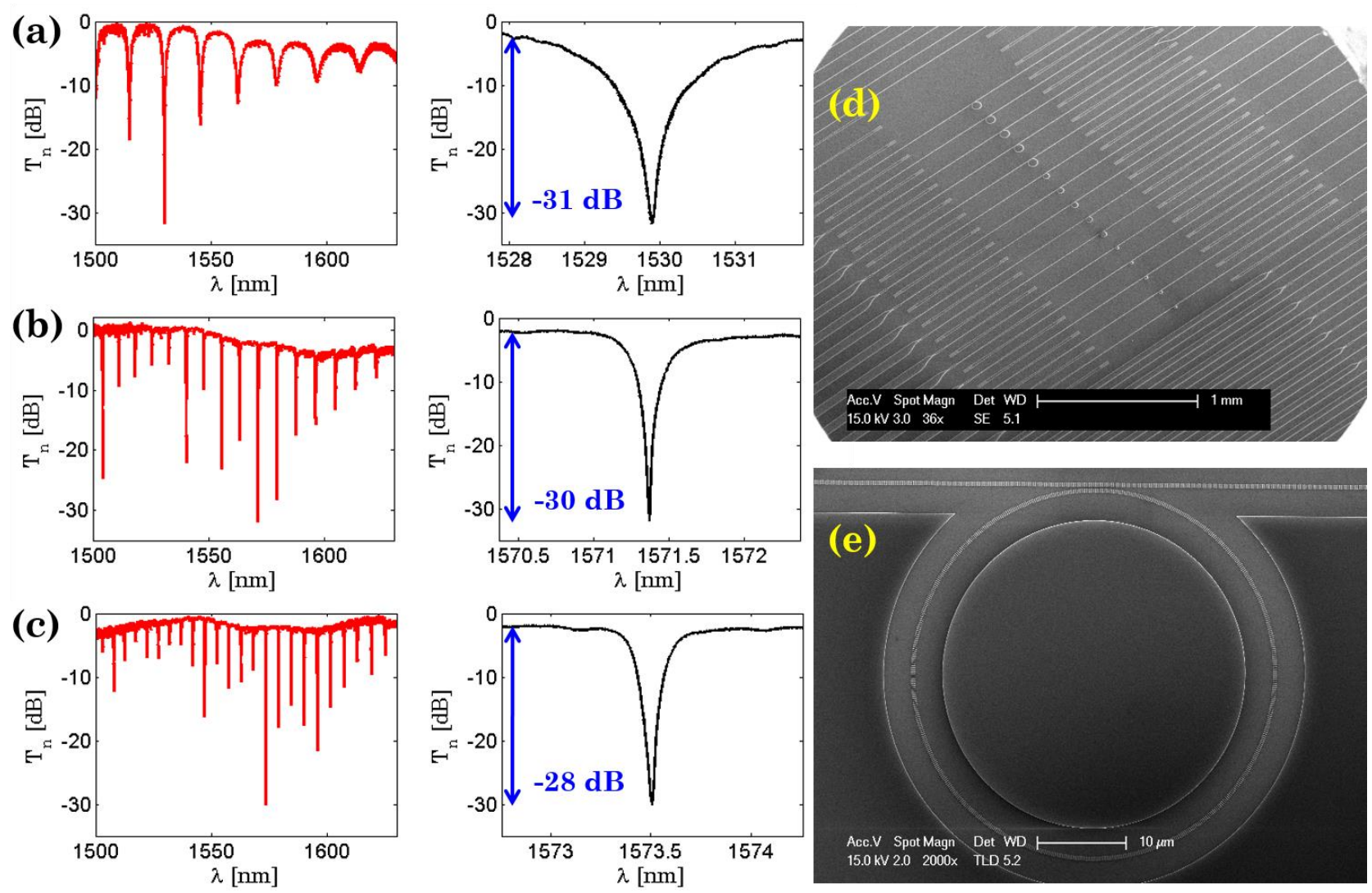

Figure 3. Spectral responses of the SWG metamaterial micro-ring waveguide resonators and the corresponding detailed resonances for ring radii of (a) $10 \mu \mathrm{m}$, (b) $20 \mu \mathrm{m}$, and (c) $30 \mu \mathrm{m}$. (d) and (e) Scanning electron microscopy images of fabricated SWG metamaterial micro-ring resonators.

\section{SILICON SUB-WAVELENGTH WAVEGUIDES WITH ENGINEERED DISPERSION}

The dispersion control in silicon nanophotonic waveguides is the key to explore a variety of emerging linear and nonlinear applications. SWG structures provide an extra degree of freedom to tailor the overall dispersion. First, in the deep SWG operation regime, the metamaterial structure does not make any contribution to the overall dispersion and spectral evolution of the equivalent metamaterial refractive index is exclusively governed by dispersion of the constituent materials. On the other hand, when the SWG period approaches the one-half of the effective wavelength in the waveguide, the metamaterial structure acts as a conventional waveguide, yet with tailorable dispersion. Up to now, only a minor works have been reported to examine the SWG metamaterial waveguide dispersion ${ }^{51,52}$.

Figures $4 \mathrm{a}$ and $4 \mathrm{~b}$ show schematics of the SWG structure considered in this work. The segmented waveguide core of SOI platform is formed by interleaving the unetched silicon blocks with a deeply etched grating trenches filled by the upper cladding material, which is silicon nitride. The detailed design process as well as various calculation approaches are described in Ref. [51]. In this work, the impact of transversal and longitudinal waveguide dimensions is investigated in detail on the evolution of the dispersion in SWG metamaterial strip waveguides.

Figures $4 \mathrm{c}-4 \mathrm{e}$ show the evolution of the dispersion within the SWG metamaterial waveguides considering various cross-sectional waveguide arrangement as well as different lengthwise geometries. For a fixed SWG waveguide 
geometry along the mode propagation, it becomes clear that metamaterial waveguides are less dispersive for thicker silicon waveguides due to the better modal confinement. The increase of silicon waveguide thickness produces a vertical upshift of the dispersion profile. In other words, the absolute dispersion of the SWG waveguides are smaller and gradually approaches the zero dispersion regions (see insets of Figs. 4d and 4e). In all studied cases, we may observe that at shorter wavelengths, the SWG waveguides support large normal dispersion profiles (negative values of waveguide dispersion). This trend is comparatively stronger for thicker silicon waveguides and for SWG structures operating nearby the Bragg reflection zone. As the wavelength increases, we may follow a very sharp evolution in the SWG waveguide dispersion. Furtheremore, in the region far away from the Bragg reflection zone, the SWG-structured waveguides operate in true SWG regime and acts as a conventional waveguide with an optically equivalent core. In this spectral range, the dispersion profiles are relatively flat across a wide range of operating wavelengths.

(a)
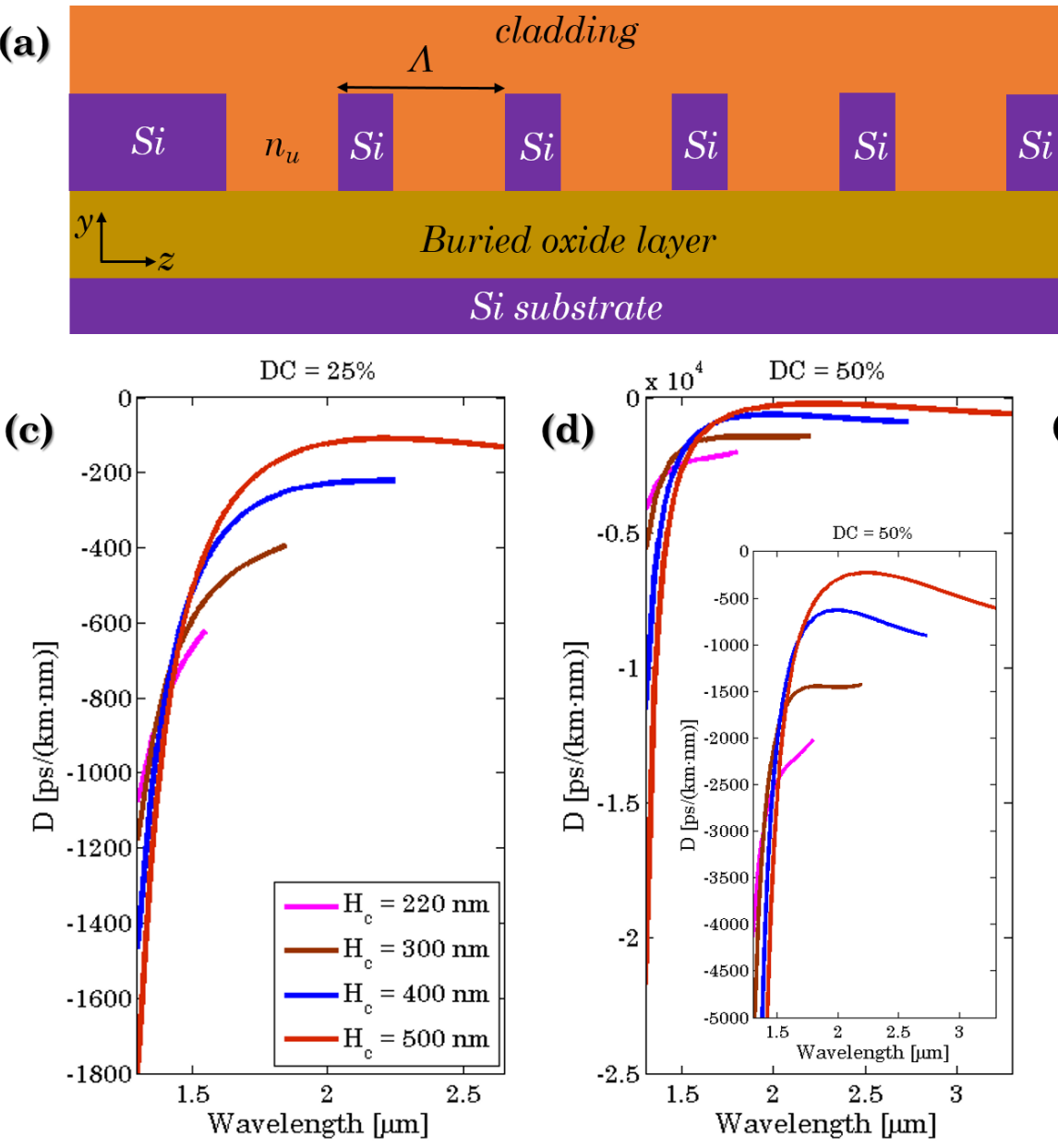
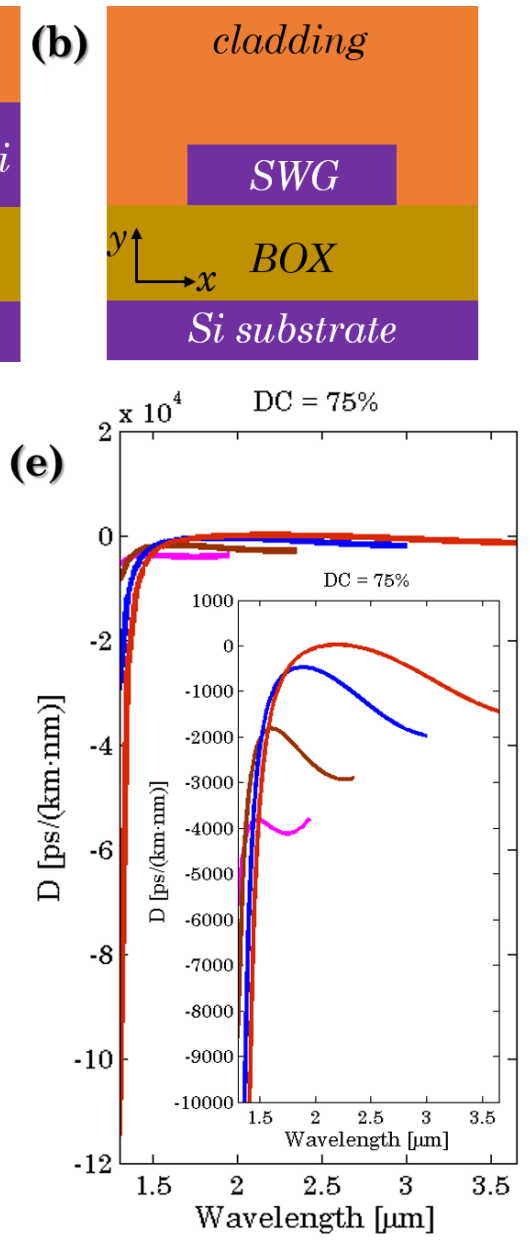

Figure 4. Schematics of the studied SWG metamaterial strip waveguides: (a) vertical and (b) transversal views. Comparative summary of the dispersion of SWG metamaterial waveguides as a function of the wavelength for various cross-sectional areas and different duty cycles of the SWG segments: (c) 25\%, (d) 50\%, and (e) 75\%. Insets: Detailed views on calculated dispersion profiles of studied SWG waveguides.

\section{CONCLUSION}

Sub-wavelength grating metamaterial waveguide structures are very attractive design tool that allows for the development of high-performing silicon nanophotonic devices and components. Available lithographic settings enable straightforward control of light propagating properties such as material and effective index, modal confinement, field profile, dispersion and anisotropy, and thereby offering an extra degree of freedom for the silicon nanophotonic designers. In this contribution, we discussed our recent achievements in sub-wavelength-engineered waveguides and devices, including low-loss fiber-chip surface grating couplers, micro-ring resonators, and dispersion-tailored strip 
metamaterial waveguides. The research work on integrated sub-wavelength grating structures progresses rapidly, with large design opportunities to be studied and explored. The complementary metal-oxide-semiconductor processings as advantageously combined with the sub-100-nm immersion lithography will most likely facilitate the mass-scale implementation of sub-wavelength silicon nanophotonics for further scientific, engineering, and even commercial exploitations.

\section{ACKNOWLEDGEMENTS}

This work was funded by European Research Council (ERC) under the European Union's Horizon 2020 Research and Innovation Program (ERC POPSTAR grant 647342).

\section{REFERENCES}

[1] L. Vivien and L. Pavesi, Handbook of Silicon Photonics (CRC Press, 2013).

[2] A. Rickman, "The commercialization of silicon photonics," Nature Photonics 8, 579-582 (2014).

[3] P. Cheben, R. Halir, J. H. Schmid, H. A. Atwater, and D. R. Smith, "Subwavelength integrated photonics," Nature 560, 565-572 (2018).

[4] R. Halir, A. Ortega-Moñux, D. Benedikovic, G. Z. Mashanovich, J. G. Wangüemert-Pérez, J. H. Schmid, Í. MolinaFernández, and P. Cheben, "Subwavelength-Grating Metamaterial Structures for Silicon Photonic Devices," Proceedings of the IEEE 106, 2144-2157 (2018).

[5] M. Berciano, G. Marcaud, P. Damas, X. Le Roux, P. Crozat, C. Alonso Ramos, D. Pérez Galacho, D. Benedikovic, D. Marris-Morini, E. Cassan, and L. Vivien, "Fast linear electro-optic effect in a centrosymmetric semiconductor ," Communications Physics 1, Article number 64, 1-9 (2018).

[6] G. T. Reed, G. Mashanovich, F. Y. Gardes, and D. J. Thomson, "Silicon optical modulators," Nature Photonics 4, 518-526 (2010).

[7] D. Marris-Morini, V. Vakarin, J. M. Ramirez, Q. Liu, A. Ballabio, J. Frigerio, M. Montesinos, C. Alonso-Ramos, X. Le Roux, S. Serna, D. Benedikovic, D. Chrastina, L. Vivien, and G. Isella, "Germanium-based integrated photonics from near- to mid-infrared applications," Nanophotonics 7, 1781-1793 (2018).

[8] R. E. Camacho-Aguillera, Y. Cai, N. Patel, J. T. Bessette, M. Romagnoli, L. C. Kimerling, and J. Michel, "Electrically pumped germanium laser," Optics Express 20, 11316-11320 (2012).

[9] D. Benedikovic, L. Virot, G. Aubin, F. Amar, B. Szelag, B. Karakus, J.-M. Hartmann, C. Alonso-Ramos, X. Le Roux, P. Crozat, E. Cassan, D. Marris-Morini, C. Baudot, F. Boeuf, J.-M. Fédéli, C. Kopp, and L. Vivien, "25 Gbps low-voltage hetero-structured silicon-germanium waveguide pin photodetectors for monolithic on-chip nanophotonic architectures," Photonics Research 7, 437-444 (2019).

[10] A. Rahim, T. Spuesens, R. Baets, and W. Bogaerts, "Open-Access Silicon Photonics: Current Status and Emerging Initiatives," Proceedings of the IEEE 106, 2313-2330 (2018).

[11] T. Komljenovic, D. Huang, P. Pintus, M. A. Tran, M. L. Davenport, and J. E. Bowers, "Silicon Integrated Circuits Using Heterogeneous Integration on Silicon," Proceedings of the IEEE 106, 2246-2257 (2018).

[12] O. Marshall, M. Hsu, Z. Wang, B. Kunert, C. Koos, and D. Van Thourhout, "Heterogeneous Integration on Silicon Photonics," Proceedings of the IEEE 106, 2258-2269 (2018).

[13] W. D. Sacher, J. C. Mikkelsen, Y. huang, J. C. C. Mak, Z. Yong, X. Luo, Y. Li, P. Dumais, J. Jiang, D. Goodwill, E. bernier, P. G.-Q. Lo, and J. K. S. Poon, "Monolithically Integrated Multilayer Silicon Nitride-on-Silicon Waveguide Platforms for 3-D Photonic Circuits and Devices," Proceedings of the IEEE 106, 2232-2245 (2018).

[14] S. Gurber, C. Alonso-Ramos, D. Benedikovic, D. Pérez-Galacho, X. Le Roux, N. Vulliet, S. Cremer, L. Babaud, J. Planchot, D. Benoit, P. Chantraine, F. Leverd, D. Ristoiu, P. Grosse, D. Marris-Morini, L. Vivien, C. Baudot, and F. Boeuf, "Integrated SiN on SOI dual photonic devices for advanced datacom solutions," Proceedings of SPIE Vol. 10686, 106860W, Silicon Photonics: From Fundamental research to Manufacturing, 1-8 (2018).

[15]P. Cheben, P. J. Bock, J. H. Schmid, J. Lapointe, S. Janz, D.-X. Xu, A. Densmore, A. Delâge, B. Lamontagne, and T. J. Hall "Refractive index engineering with subwavelength gratings for efficient microphotonic couplers and planar waveguide multiplexers," Optics Letters 35, 2526-2528 (2010). 
[16]P. J. Bock, P. Cheben, J. H. Schmid, J. Lapointe, A. Delâge, S. Janz, G. C. Aers, D.-X. Xu, A. Densmore, and T. J. Hall, "Subwavelength grating periodic structures in silicon-on-insulator: A new type of microphotonic waveguide," Optics Express 18, 20251-20262 (2010).

[17] S. M. Rytov, "Electromagnetic properties of a finely stratified medium," Sov. Phys. JETP 2, 466-475 (1956).

[18] R. Halir, P. Cheben, J.-M. Luque-González, J.-D. Sarmiento-Merenguel, J. H. Schmid, G. Wangüemert-Pérez, D.-X. Xu, S. Wang, A. Ortega-Moñux, and Í. Molina-Fernández, "Ultra-broadband nanophotonic beamsplitter using an anisotropic sub-wavelength metamaterial," Laser \& Photonics Reviews 10, 1039-1046 (2016).

[19] J.-M. Luque-González, A. Herrero-Bermello, A. Ortega-Moñux, Í. Molina-Fernández, A. V. Velasco, P. Cheben, J. H. Schmid, S. Wang, and R. Halir, "Tilted subwavelength gratings: controlling anisotropy in metamaterial nanophotonic waveguides," Optics Letters 43, 4691-4694 (2018).

[20] B. Peng, C. Xiong, M. Khater, A. S. Jensen, W. M. J. Green, and T. Barwicz, "Metamaterial waveguides with low distributed backscattering in production O-band Si photonics," Proceedings of in Optical Fiber Communication Conference (OFC, 2015), paper Tu3K.3.

[21] J. D. Sarmiento-Merenguel, A. Ortega-Moñux, J.-M. Fédéli, J. G. Wangüemert-Pérez, C. Alonso-Ramos, E. DuránValdeiglesias, P. Cheben, Í. Molina-Fernández, and Robert Halir, "Controlling leakage losses in subwavelength grating silicon metamaterial waveguides," Optics Letters 41, 3443-3446 (2016).

[22] A. Ortega-Moñux, J. Čtyroký, P. Cheben, J. H. Schmid, S. Wang, Í. Molina-Fernández, and R. Halir, "Disorder effects in subwavelength grating metamaterial waveguides," Optics Express 25, 12222-12236 (2017).

[23] P. Cheben, D.-X. Xu, S. Janz, and A. Densmore, "Subwavelength waveguide grating for mode conversion and light coupling in integrated optics," Optics Express 14, 4695-4702 (2006).

[24] T. Barwicz, A. Janta-Polczynski, M. Khater, Y. Thibodeau, R. Leidy, J. Maling, S. Martel, S. Engelman, J. S. Orcutt, P. Fortier, and W. M. J. Green, "An O-band metamaterial converter interfacing standard optical fibers to silicon nanophotonics waveguides," Proceedings of in Optical Fiber Communication Conference (OFC, 2015), paper Th3F.3.

[25]P. Cheben, J. H. Schmid, S. Wang, D.-X. Xu, M. Vachon, S. Janz, J. Lapointe, Y. Painchaud, and M.-J. Picard, "Broadband polarization independent nanophotonic coupler for silicon waveguides with ultra-high efficiency," Optics Express 23, 22553-22563 (2015).

[26] M. Papes, P. Cheben, D. Benedikovic, J. H. Schmid, J. Pond, R. Halir, A. Ortega-Moñux, G. Wangüemert-Pérez, W. N. Ye, D.-X. Xu, S. Janz, M. Dado, and V. Vasinek, "Fiber-chip edge coupler with large mode size for silicon photonic wire waveguides," Optics Express 24, 5026-5038 (2016).

[27] R. Halir, P. Cheben, S. Janz, D.-X. Xu, I. Molina-Fernández, and J.-G. Wangüemert-Pérez, "Waveguide grating coupler with subwavelength microstructures," Optics Letters 34, 1408-1410 (2009).

[28] R. Halir, P. Cheben, J. H. Schmid, R. Ma, D. Bedard, S. Janz, D.-X. Xu, A. Densmore, J. Lapointe, and I. MolinaFernández, "Continuously apodized fiber-to-chip surface grating coupler with refractive index engineered subwavelength structure," Optics Letters 35, 3243-3245 (2010).

[29] X. Chen and H. K. Tsang, "Nanoholes grating couplers for coupling between silicon-on-insulator waveguides and optical fibers," IEEE Photonics Journal 1, 184-190 (2009).

[30] D. Benedikovic, P. Cheben, J. H. Schmid, D.-X. Xu, J. Lapointe, S. Wang, R. Halir, A. Ortega-Moñux, S. Janz, and M. Dado, "High-efficiency single etch step apodized surface grating coupler using subwavelength structure," Laser \& Photonics Reviews 8, 93-97 (2014).

[31] Y. Ding, C. Peucheret, H. Ou, and K. Yvind, "Fully etched apodized grating coupler on the SOI platform with -0.58 dB coupling efficiency," Optics Letters 39, 5348-5350 (2014).

[32] D. Benedikovic, P. Cheben, J. H. Schmid, D.-X. Xu, B. Lamontagne, S. Wang, J. Lapointe, R. Halir, A. OrtegaMoñux, S. Janz, and M. Dado, "Subwavelength index engineered surface grating coupler with sub-decibel efficiency for 220-nm silicon-on-insulator waveguides," Optics Express 23, 22628-22635 (2015).

[33] D. Benedikovic, C. Alonso-Ramos, P. Cheben, J. H. Schmid, S. Wang, R. Halir, A. Ortega-Moñux, D.-X. Xu, L. Vivien, J. Lapointe, S. Janz, and M. Dado, "Single-etch subwavelength engineered fiber-chip grating couplers for $1.3 \mu \mathrm{m}$ datacom wavelength band," Optics Express 24, 12893-12904 (2016).

[34] C. Alonso-Ramos, P. Cheben, A. Ortega-Moñux, J. H. Schmid, D.-X. Xu, and I. Molina-Fernández, "Fiber-chip grating coupler based on interleaved trenches with directionality exceeding 95\%," Optics Letters 39, 5351-5354 (2014).

[35] D. Benedikovic, C. Alonso-Ramos, P. Cheben, J. H. Schmid, S. Wang, D.-X. Xu, J. Lapointe, S. Janz, R. Halir, A. Ortega-Moñux, J. G. Wangüemert-Pérez, I. Molina-Fernández, J.-M. Fédéli, L. Vivien, and M. Dado, "High- 
directionality fiber-chip grating coupler with interleaved trenches and subwavelength index-matching structure," Optics Letters 40, 4190-4193 (2015).

[36] X. Chen, D. J. Thomson, L. Crudginton, A. Z. Khokhar, and G. T. Reed, "Dual-etch apodised grating couplers for efficient fibre-chip coupling near $1310 \mathrm{~nm}$ wavelength," Optics Express 25, 17864-17871 (2017).

[37] D. Benedikovic, C. Alonso-Ramos, D. Pérez-Galacho, S. Guerber, V. Vakarin, G. Marcaud, X. Le Roux, E. Cassan, D. Marris-Morini, P. Cheben, F. Boeuf, C. Baudot, and L. Vivien, "L-shaped fiber-chip grating couplers with high directionality and low reflectivity fabricated with deep-UV lithography," Optics Letters 42, 3439-3442 (2017).

[38] T. Watanabe, M. Ayata, U. Koch, Y. Fedoryshyn, and J. Leuthold, "Perpendicular Grating Coupler Based on a Blazed Antiback-Reflection Structure,” IEEE/OSA Journal of Lightwave Technology 35, 4663-4669 (2017).

[39] R. Halir, A. Maese-Novo, A. Ortega-Moñux, I. Molina-Fernández, J. G. Wangüemert-Pérez, P. Cheben, D.-X. Xu, J. H. Schmid, and S. Janz, "Colorless directional coupler with dispersion engineered sub-wavelength structure," Optics Express 20, 13470-13477 (2012).

[40]H. Yun, Y. Wang, F. Zhang, Z. Lu, S. Lin, L. Chrostowski, and N. A. F. Jaeger, "Broadband $2 \times 2$ adiabatic $3 \mathrm{~dB}$ coupler using silicon-on-insulator sub-wavelength grating waveguides," Optics Letters 41, 3041-3044 (2016).

[41] D. Pérez-Galacho, C. Alonso-Ramos, F. Mazeas, X. Le Roux, D. Oser, W. Zhang, D. Marris-Morini, L. Labonté, S. Tanzilli, É. Cassan, and L. Vivien, "Optical pump-rejection filter based on silicon sub-wavelength engineered photonic structures," Optics Letters 42, 1468-1471 (2017).

[42] D. Oser, D. Pérez-Galacho, C. Alonso-Ramos, X. Le Roux, S. Tanzilli, L. Vivien, L. Labonté, and É. Cassan, "Subwavelength engineering and asymmetry: two efficient tools for sub-nanometer-bandwidth silicon Bragg filters," Optics Letters 43, 3208-3211 (2018).

[43] J. Čtyroký, J. G. Wangüemert-Pérez, P. Kwiecien, I. Richter, J. Litvik, J. H. Schmid, Í. Molina-Fernández, A. Ortega-Moñux, M. Dado, and P. Cheben, "Design of narrowband Bragg spectral filters in subwavelength grating metamaterial waveguides," Optics Express 26, 179-194 (2018).

[44]P. Cheben, J. Čtyroký, J. H. Schmid, S. Wang, J. Lapointe, J. G. Wangüemert-Pérez, Í. Molina-Fernández, A. Ortega-Moñux, R. Halir, D. Melati, D.-X. Xu, S. Janz, and M. Dado, "Bragg filter bandwidth engineering in subwavelength grating metamaterial waveguides," Optics Letters 44, 1043-1046 (2019).

[45] J. G. Wangüemert-Pérez, P. Cheben, A. Ortega-Moñux, C. Alonso-Ramos, D. Pérez-Galacho, R. Halir, I. MolinaFernández, D.-X. Xu, and J. H. Schmid, "Evanescent field waveguide sensing with subwavelength grating structures in silicon-on-insulator," Optics Letters 39, 4442-4445 (2014).

[46] V. Donzella, A. Sherwali, J. Flueckiger, S. M. Grist, S. T. Fard, and L. Chrostowski, "Design and fabrication of SOI micro-ring resonators based on sub-wavelength grating waveguides," Optics Express 23, 4791-4803 (2015).

[47] J. Flueckiger, S. Schmidt, V. Donzella, A. Sherwali, D. M. Ratner, L. Chrostowski, and K. C. Cheung, "Subwavelength grating for enhanced ring resonator biosensor," Optics Express 24, 15672-15686 (2016).

[48] H. Yan, L. Huang, X. Xu, S. Chakravarty, N. Tang, H. Tian, and R. T. Chen, "Unique surface sensing property and enhanced sensitivity in microring resonator biosensors based on subwavelength grating waveguides," Optics Express 24, 29724-29733 (2016).

[49] L. Huang, H. Yan, X. Xu, S. Chakravarty, N. Tang, H. Tian, and R. T. Chen, "Improving the detection limit for onchip photonic sensors based on subwavelength grating racetrack resonators," Optics Express 25, 10527-10535 (2017).

[50]Z. Wang, X. Xu, D. Fan, Y. Wang, and R. T. Chen, "High quality factor subwavelength grating waveguide microring resonator based on trapezoidal silicon pillars," Optics Letters 41, 3375-3378 (2016).

[51]D. Benedikovic, M. Berciano, C. Alonso-Ramos, X. Le Roux, E. Cassan, D. Marris-Morini, and L. Vivien, "Dispersion control of silicon nanophotonic waveguides using sub-wavelength grating metamaterials in near- and mid-IR wavelengths," Optics Express 25, 19468-19478 (2017).

[52]Z. Jafari and A. Zarifkar, "Dispersion flattened single etch-step waveguide based on subwavelength grating," Optics Communications 393, 219-223 (2017).

[53]Z. Pan, X. Xu, C.-J. Chung, H. Dalir, H. Yan, K. Chen, Y. Wang, B. Jia, and R. T. Chen, "High-Speed Modulator Based on Electro-Optic Polymer Infiltrated Subwavelength Grating Waveguide Ring resonator," Laser \& Photonics Reviews 12, 1-6 (2018).

[54] J. Soler Penades, A. Ortega-Moñux, M. Nedeljkovic, J. G. Wangüemert-Pérez, R. Halir, A. Z. Khokhar, C. AlonsoRamos, Z. Qu, I. Molina-Fernández, P. Cheben, and G. Z. Mashanovich, "Suspended silicon mid-infrared waveguide devices with subwavelength grating metamaterial cladding," Optics Express 24, 22908-22916 (2016).

[55] J. Soler Penadés, A. Sánchez-Postigo, M. Nedeljkovic, A. Ortega-Moñux, J. G. Wangüemert-Pérez, Y. Xu, R. Halir, Z. Qu, A. Z. Khokhar, A. Osman, W. Cao, C. G. Littlejohns, P. Cheben, I. Molina-Fernández, and G. Z. 
Mashanovich, "Suspended silicon waveguides for long-wave infrared wavelengths," Optics Letters 43, 795-798 (2018).

[56] A. Osman, M. Nedeljkovic, J. Soler Penades, Y. Wu, Z. Qu, A. Z. Khokhar, K. Debnath, and G. Z. Mashanovich, "Suspended low-loss germanium waveguides for the longwave infrared," Optics Letters 43, 5997-6000 (2018).

[57]R. Halir, P. Bock, P. Cheben, A. Ortega-Moñux, C. Alonso-Ramos, J. H. Schmid, J. Lapointe, D.-X. Xu, J. G. Wangüemert-Pérez, I. Molina-Fernández, and S. Janz, "Waveguide sub-wavelength structures: a review of principles and applications," Laser \& Photonics Reviews 9, 25-49 (2015). 